\title{
Consequences of Dust in Metal-Rich H II Regions
}

\author{
Joseph C. Shields ${ }^{1}$ and Robert C. Kennicutt, Jr. \\ Steward Observatory, University of Arizona, Tucson, AZ 85721
}

\begin{abstract}
Dust and associated depletion of heavy elements from the gas phase can modify the thermal properties of H II regions from the dust-free case, with significant consequences for the emergent optical spectrum. We present the results of theoretical calculations illustrating the effects of grains on the spectra of giant, extragalactic H II regions, with emphasis on high metallicity systems $\left(Z \gtrsim Z_{\odot}\right)$. Dust provides a simple explanation for the observational absence of pure Balmer-line spectra that are expected on theoretical grounds for dust-free, chemically enriched nebulae. Grains may also play a role in enhancements of forbidden-line emission observed in H II regions in the enriched nuclei of normal galaxies. In most cases, depletion introduces the strongest perturbations to the optical spectrum. Selective absorption of the ionizing continuum as well as heating by grain photoelectrons are important in some instances, however, and grain heating can be particularly important for enhancing emission in high-ionization lines. Allowing for depletion, the presence of dust is unlikely to introduce large errors in global metallicity indicators, although uncertainties in depletion factors coupled with the sensitivity of infrared cooling to electron density will make accurate calibrations difficult at high $Z$. The present calculations establish further that previous relative abundance analyses that fail to take into account dust effects in a self-consistent way (grain heating as well as depletion) may overestimate temperature gradients in high- $Z$ nebulae, resulting in errors in relative abundances for different elements.
\end{abstract}

Subject headings: ISM: H II Regions - ISM: Dust - ISM: Abundances

\section{Introduction}

Giant H II regions have a fundamental role as probes of elemental abundances in extragalactic environments. Existing spectroscopic studies of extragalactic H II regions tend to emphasize systems with subsolar metallicities, due in part to inherent interest in chemically unevolved systems, but also because of selection effects, since characteristic luminosities and emission-line

\footnotetext{
${ }^{1}$ Hubble Fellow
} 
equivalent widths for HII regions tend to decrease with increasing source metallicity (e.g., Kennicutt 1988). Data for nebulae in relatively high-metallicity environments, such as the inner regions of giant galaxies, are becoming increasingly available, however, and additional theoretical work is necessary if we are to make use of this information for quantitative abundance analyses.

The presence of abundant atomic coolants in enriched H II regions will typically lead to a low equilibrium electron temperature, with a large fraction of the radiated energy emitted by the heavy elements through infrared ground-state fine structure transitions. The strengths of the comparatively energetic optical lines are very sensitive to the electron temperature, and hence to nebular parameters that affect temperature. At abundances above a few times solar, the fine-structure cooling is efficient enough that optical forbidden lines from simple, low-density H II regions are expected to be unobservable (e.g., Rubin 1985). To date, however, no H II region emitting a pure Balmer-line spectrum has been discovered, even in surveys of $\mathrm{H} \alpha$-selected H II regions in environments for which metallicity $Z \gg \mathrm{Z}_{\odot}$ is expected (Oey \& Kennicutt 1993). The lack of such objects implies that physical parameters in real nebulae are modified so that the electron temperature is high enough to produce significant optical forbidden-line emission.

The effects of interstellar dust could conceivably underlie at least some of the enhancement of optical emission in high- $Z$ H II regions. Dust can act to modify the thermal structure of a nebula in several ways. The removal of atomic coolants from the gas phase via depletion will tend to increase the electron temperature, leading to measurable changes in the emitted spectrum (e.g., Henry 1993). Grains may selectively absorb a fraction of the Lyman continuum photons powering the nebula, resulting in modification to the ionizing spectral energy distribution seen by the gas and to the resultant heating (e.g., Sarazin 1977; Mathis 1986; Aannestad 1989). Grains will also participate directly in the thermal balance by contributing photoelectrons that heat the plasma, and by cooling through radiation of energy transferred in captures of electrons (e.g., Oliveira \& Maciel 1986; Baldwin et al. 1991).

Previous studies emphasizing nebulae at $Z \lesssim \mathrm{Z}_{\odot}$ have generally concluded that selective absorption of the ionizing continuum by dust has a relatively small effect on the emergent optical spectrum of diffuse H II regions (e.g., Mathis 1986). Given the greater temperature sensitivity of optical lines at higher $Z$, we might expect the influence of dust to be more important in metal-rich nebulae; this statement is especially true if the dust-to-gas ratio scales with $Z$. The observational consequences of grain heating and cooling within H II regions over the entire span of metallicity have also received only limited attention in previous work.

Motivated by these considerations, we have undertaken photoionization calculations appropriate for giant extragalactic H II regions in order to understand possible consequences of dust in chemically enriched nebulae. We find that dust and associated depletion can indeed produce nonnegligible perturbations in the optical spectrum of metal-rich H II regions. The influence of dust in such objects provides a possible basis for explaining several unexpected empirical characteristics of H II regions in high- $Z$ environments, including the lack of pure 
Balmer-line nebulae. If grains survive in the inner parts of spherical nebulae, they may selectively enhance emission in high-ionization lines. Dust is unlikely to contribute large uncertainties in gas phase abundance estimates based on the oxygen emission lines, but ambiguities in depletion factors and density effects will contribute substantial uncertainty to "empirical" metallicity-excitation calibrations for $Z \gtrsim \mathrm{Z}_{\odot}$ (e.g., Pagel et al. 1979). Finally, we suggest possible revisions to theoretical relations between ion-weighted temperatures employed in relative abundance analyses, necessitated by a self-consistent treatment of the presence of dust in H II regions.

\section{Nebular Calculations with Dust}

Calculations appropriate for prediction of H II region optical spectra were generated with the photoionization code Cloudy, version 84.09 (Ferland 1994). Parameters were chosen such that the results would illustrate the possible consequences of dust in giant, extragalactic H II regions (GEHRs) as a function of metallicity. The ionizing continuum was represented by Kurucz (1979) stellar atmospheres corresponding to $T_{\star}$ of $38,000 \mathrm{~K}$ and $45,000 \mathrm{~K}$, with total ionizing luminosities (photons s ${ }^{-1}$ ) of $\log Q(\mathrm{H})=50.7$ and 51.2 , respectively. The cooler stellar continuum model is interpolated from theoretical calculations for $T_{\star}=35,000 \mathrm{~K}$ and 40,000 K, and both of the final continuum choices assume stellar $\log (g)=4.5$. Based on systematic trends in existing observations, the lower $T_{\star}$ values are more applicable for nebulae with relatively high $Z$ (e.g., McCall, Rybski, \& Shields 1985; Vílchez \& Pagel 1988).

The nebular geometry was assumed to be spherical, extending from an inner radius of 0.01 pc to the Strömgren radius $R_{s}$ defined as the point where the ionized hydrogen fraction fell below 1\%. A total hydrogen density of $n_{H}=10 \mathrm{~cm}^{-3}$ was adopted for material described by volume filling factors of $\epsilon=0.0617$ and 0.0347 for the $38,000 \mathrm{~K}$ and $45,000 \mathrm{~K}$ cases, respectively. If we define ionization parameter $U$ in the customary way $\left[U \equiv Q(\mathrm{H}) /\left(4 \pi R_{s}^{2} n_{e} c\right)\right.$, where $c$ is the speed of light], these combinations of parameters yield $\log U=-3.0$ at $Z=\mathrm{Z}_{\odot}$ in the dust-free case; estimates of $U$ for GEHRs are comparable to or somewhat larger than this value (Shields 1990 and references therein). $U$ is a function of $Z$, due to the dependence of recombination rates and hence $R_{s}$ on electron temperature $T_{e}$, as well as a function of dust content, since grains will absorb a portion of the ionizing radiation and also modify the $T_{e}$ structure of the nebula through heating and cooling processes.

Total elemental abundances for the case of $Z=Z_{\odot}$ were taken from Grevesse \& Anders (1989). For other values of $Z$, abundances of elements heavier than helium were assumed to be in solar proportions, with the exception of nitrogen, which was assumed to have a secondary component such that its abundance scales as $Z^{2}$. Empirical evidence for a secondary contribution to $\mathrm{N}$ in GEHRs is discussed, for example, in Vila-Costas \& Edmunds (1993). Depletion onto grains was assumed to modify gas-phase abundances by factors taken from Cowie \& Songaila (1986, 
Table 3) corresponding to an overall average for the interstellar medium (ISM). The resulting total and depleted abundances at $Z=\mathrm{Z}_{\odot}$ are listed in Table 1 .

The grains themselves were assumed to consist of a graphite-silicate mix with a size distribution as described by Mathis, Rumpl, \& Nordsieck (1977) for the diffuse ISM, with optical constants as given by Draine \& Lee (1984) and extended within the extreme ultraviolet (EUV) bandpass by Martin \& Rouleau (1989). The resulting dust opacity features a maximum at photon energies of $\sim 17 \mathrm{eV}$ and a decrease at higher energies, as required by the Kramers-Kronig relations for realistic grain materials (e.g., Bohren \& Huffman 1983; Mathis 1986).

Scattering by grains is neglected in the present calculations. At ionizing energies, the scattering phase function for the grains is expected to be predominantly forward-throwing. At optical wavelengths, scattering in and out of the line of sight will approximately cancel in a spherical geometry. In the current implementation of Cloudy, reddening of the emergent spectrum by grain absorption is also neglected in the spherical case. Our principal interest in the present study is the consequences of dust for the structural and thermal characteristics of nebulae, rather than on emission-line transfer effects of the dust. The effects of internal scattering and absorption have been discussed previously by Mathis (1970, 1983), Petrosian \& Dana (1980), and Aannestad (1989), among others. For the cases of interest here, the scattering and absorption optical depths within $R_{s}$ are relatively small (\$4.3.); in such cases, dereddening of the observed optical spectrum based on the Balmer line strengths is expected to reproduce the intrinsic line ratios with reasonable accuracy (Mathis 1983). As a result, the predictions of line ratios presented here are suitable for direct comparison with dereddened ratios derived from observations.

Thermal effects of dust are quantified by extending the calculation of nebular ionization and energy equilibrium to include grains. Additional details concerning the treatment of grain physics within Cloudy can be found in Baldwin et al. (1991, Appendix C) and Ferland (1994). The dust-to-gas ratio was assumed to scale in proportion with $Z$, with a normalization of 0.01 by mass at $Z=\mathrm{Z}_{\odot}$.

Giant H II regions exhibit supersonic line widths that correlate in amplitude with emission-line luminosity (Melnick 1979). Line widths in excess of thermal values will modify the transfer of lines including infrared fine-structure transitions that would otherwise be optically thick in a quiescent nebula. The added velocity width facilitates the escape of these lines, and thus may decrease the equilibrium $T_{e}$ when the infrared transitions contribute a significant fraction of the total cooling (e.g., Simpson 1975). We consequently assumed the presence of a microturbulent velocity component $b_{t u r b}$ added in quadrature with the thermal $b\left(\equiv\left[2 k T_{e} / m\right]^{0.5}\right)$, with values of $b_{\text {turb }}=17.4 \mathrm{~km} \mathrm{~s}^{-1}$ for the case of $T_{\star}=38,000 \mathrm{~K}$ and $\log Q(\mathrm{H})=50.7$, and $b_{\text {turb }}=23.0 \mathrm{~km} \mathrm{~s}^{-1}$ for $T_{\star}=45,000 \mathrm{~K}$ and $\log Q(\mathrm{H})=51.2$. These choices of $b_{\text {turb }}$ are derived from the empirical relation between recombination line luminosity and velocity width given by Arsenault \& Roy (1988). 


\section{General Results}

The predicted strengths of optical forbidden lines, normalized to hydrogen recombination features, are plotted as a function of $Z$ in Figures 1 and 2 for $T_{\star}=38,000 \mathrm{~K}$ and 45,000 K, respectively. The curves show the expected behavior with increasing $Z$, with forbidden-line emission initially rising in response to the growing number of emitters, reaching a maximum, and declining at high $Z$ as the total cooling efficiency increases and $T_{e}$ diminishes. The ratio of [O II] $\lambda 3727 / \mathrm{H} \beta$ exhibits an additional reversal, with increasing values at very high $Z$. This behavior stems from photoionization of $\mathrm{O}^{0}$ into excited states of $\mathrm{O}^{+}$that can subsequently decay radiatively. The resulting emission component consequently scales directly with the abundance of $\mathrm{O}$ and hence with $Z$. While such emission can be important in some contexts (e.g., novae; Ferland \& Truran 1981), it is unlikely to be significant in H II regions, for which $[\mathrm{O}$ II $] / \mathrm{H} \beta \gtrsim 0.3$ observationally (e.g., Oey \& Kennicutt 1993).

The separate curves in a given plot in Figures 1 and 2 illustrate the change in emission line behavior as the effects of dust are added incrementally. The solid curve corresponds to dust-free, undepleted nebulosity, while the other curves show the results of introducing depletion, dust opacity, and grain heating + cooling. In almost all cases, the effects of dust lead to an enhancement of optical forbidden-line emission over the undepleted, dust-free case, due to an increase in equilibrium $T_{e}$.

For most of the lines, the largest shift in the plotted line ratio curves results when depletion is introduced. This pattern implies that depletion of gas-phase coolants is likely to be the most important aspect of dust in terms of its influence on the observed spectrum (see also Mathis 1986); this statement is supported by comparisons of how forbidden line strengths change when the three effects of dust are added in isolation, rather than in succession. While depletion of optically emitting elements can produce a reduction of line emission at low $Z$, the result is more typically an increase in optical line emission as the cooling efficiency of other transitions decreases and equilibrium $T_{e}$ increases. Infrared fine-structure transitions are particularly important for establishing this behavior, since they scale in strength with abundance but are relatively insensitive to $T_{e}$. Henry (1993) has emphasized the importance of the [Si II] $34.8 \mu \mathrm{m}$ line and infrared [Fe II] transitions for cooling in an undepleted nebula; in real nebulae, these elements are likely to be subject to significant depletion onto grains, producing an increase in $T_{e}$ that can boost the strength of optical lines.

Selective absorption of ionizing radiation by dust additionally modifies the ionization and thermal structure of a nebula, leading to changes in its optical spectrum. The decrease in grain opacity at photon energies above the Lyman limit results in a hardening of the ionizing radiation field as it is attenuated by grains. The resultant boost in average photon energy produces greater heating per ionization, with a corresponding increase in $T_{e}$ and optical line emission. The amplitude of this effect in GEHRs is likely to be small, based on the results in Figures 1 and 2 as well as analytic arguments. The optical depth of dust will scale with the total column density of 
gas within the Strömgren radius, $N_{H}=n_{H} R_{s}$, and the dust-to-gas ratio, assumed here to scale with $Z$. From the definition of $U$, we can express

$$
N_{H}=U \frac{3 c}{\alpha_{B}} \approx 3 \times 10^{20}\left(\frac{U}{10^{-3}}\right) \mathrm{cm}^{-2}
$$

where the case B hydrogen recombination coefficient $\alpha_{B}$ for $T_{e}=7500 \mathrm{~K}$ (approximately correct for $Z=\mathrm{Z}_{\odot}$ ) and $n_{e}=100 \mathrm{~cm}^{-3}$ is taken from Hummer \& Storey (1987). For a dust-to-gas ratio representative of the Galactic ISM, the maximum EUV absorption optical depth will occur at approximately $17 \mathrm{eV}$, with $\tau_{E U V} \approx\left(2 \times 10^{-21} \mathrm{~cm}^{2}\right) N_{H}$ (Martin \& Rouleau 1989). For a Strömgren sphere, the maximum EUV optical depth with thus be

$$
\tau_{E U V} \approx 0.6\left(\frac{U}{10^{-3}}\right)\left(\frac{Z}{\mathrm{Z}_{\odot}}\right)
$$

where $U$ is defined in the dust-free limit. The expected $\tau_{E U V}$ in GEHRs is thus significant for typical parameters, and will be higher if the nebulae are relatively dusty and/or $U$ is elevated above typical values. Substantial modification of the ionizing spectral energy distribution is more likely in Galactic ultracompact and blister H II regions (e.g., Aannestad 1989), which may be described by $U$ values much greater than those typical of GEHRs. When dust opacity is large, $R_{s}$ and $\tau_{E U V}$ are obviously coupled further by the diminution of ionizing flux seen by atomic material, a point considered in more detail in $\$ 4.3$.

Figures 1 and 2 also demonstrate that grain heating boosts the predicted optical emission even further. Photoelectrons emitted by grains are expected to be more energetic and hence produce greater heating than photoelectrons emitted by the constituent atoms in isolation, because the ionization potential, or work function, is reduced for matter in a solid phase (e.g., see Draine 1989 for a review). The treatment of grains employed by Cloudy assumes a minimum work function of $8 \mathrm{eV}$ for a neutral grain, with an increase in threshold energy as the grain becomes more strongly depleted of electrons and charged. The heating rate scales directly with the density of grains and, for a weak grain potential, the flux of radiation above the threshold energy. In a dust-free nebula, heating tends to be dominated by photoelectrons from recombined hydrogen and hence scales as $n_{e}^{2}$. For an H II region with fixed density and dust-to-gas ratio, the significance of grain heating relative to hydrogen bound-free heating consequently scales with the flux $\phi$ of energetic radiation (or more generally, with ionization parameter $U$, defined locally as a ratio of ionizing photon to electron densities). The relative dependence of grain heating on $\phi$ introduces a radial variation in the resulting thermal effects for a spherical nebula, with greater heating occurring at small radii (see $\$$ 5.2.). Grain cooling by electron attachment is also likely to be significant, and the relative importance of heating and cooling by grains is a more complicated function of the ionizing spectral energy distribution, grain potential, and $T_{e}$ (Baldwin et al. 1991, Appendix C.14).

These initial results suggest that the collective effects of dust may indeed account for the absence of observed pure Balmer-line nebulae. While [O III] emission may become unobservable in low excitation GEHRs, [O II] emission is invariably detected, as noted previously, with [O II] 
$\lambda 3727 / \mathrm{H} \beta \gtrsim 0.3$. This minimum is consistent in the present calculations with $Z$ as high as $2-3 \times \mathrm{Z}_{\odot}$. Observed strengths of $[\mathrm{N} \mathrm{II}]$ and $[\mathrm{S} \mathrm{II}]$ are similarly consistent in general terms with metallicities as high as this bound.

\section{Verisimilitude}

\subsection{Grain Assumptions}

The calculations presented here make a number of assumptions of uncertain validity for GEHRs. The greatest uncertainty in this discussion is the detailed nature of grains in these nebulae. From studies of the Galactic ISM, it is well established that depletion rates are a function of environment (Cowie \& Songaila 1986 and references therein). Given the substantial output of radiative and kinetic energy by young stars, grains located near these sources might be expected to be modified and/or partially destroyed. Strom et al. (1974) presented a comparison of infrared emission from Galactic and extragalactic HII regions that has been interpreted as evidence for grain destruction in the GEHRs. Galactic sources exhibit a correlation between thermal radio continuum emission and infrared luminosity that Strom et al. employed to predict infrared fluxes from a group of more luminous GEHRs, using $\mathrm{H} \beta$ emission in place of radio continuum emission to trace $Q(\mathrm{H})$. Observed $10-20 \mu \mathrm{m}$ fluxes for the extragalactic sources fall short of the predicted fluxes derived in this manner. Strom et al. discussed several possible explanations for this discrepancy, including a deficiency of dust in the luminous extragalactic sources.

The difference noted by Strom et al. between measured IR fluxes and the extrapolated predictions may be a manifestation of a more general pattern of disparity between high- and low-luminosity sources. As discussed by Habing \& Israe (1979) and Kennicutt (1984), H II regions show systematic trends with luminosity such that lower luminosity sources are denser and more centrally concentrated than their giant counterparts. Both tendencies might be expected to result in higher $U$ for the Galactic sources, and consequently greater heating of dust grains and resultant IR emission, consistent with the observational trend found by Strom et al. Alternatively, studies of the Galactic ISM demonstrate that depletion and hence the dust-to-gas ratio correlate with density (Mathis 1990), which would be consistent with a higher grain content in dense Galactic H II regions when compared with more diffuse GEHRs.

Recent clues to the grain content of GEHRs are provided by analyses of silicon and iron abundances in H II regions and compact blue galaxies by Garnett et al. (1995) and Thuan (1995, private communication). In both studies the results suggest that the depletion rates of these elements are lower in GEHRs than in the average ISM. These findings support a picture in which a fraction of the grains in nebulae are destroyed, such that the dust-to-gas ratio in GEHRs is reduced somewhat from a simple extrapolation based on the Galactic ISM. If these results are 
characteristic of GEHRs, the nebular predictions described here will exaggerate to some degree the actual effects of dust in these sources.

The present calculations also assume a specific grain size distribution, which is again known to be a function of environment. The EUV grain opacity and rate of grain heating are very sensitive to the small grain component; if the small grain population is reduced compared to the ISM average, as inferred in the Orion nebula and other dense environs (Mathis 1990 and references therein), the effects of dust opacity and heating will be correspondingly diminished. If 30 Doradus can be regarded as representative of GEHRs, however, the small grain component may actually be enhanced relative to grains in the Galactic ISM (Fitzpatrick 1986). The elemental content of dust in GEHRs is a further major uncertainty. The relative depletion rates are poorly constrained, and more generally, the total relative abundances may differ from a simple scaling of solar proportions. In light of these many fundamental ambiguities, the present calculations can only be considered illustrative of the effects of dust in H II regions, rather than providing definitive predictions.

\subsection{Line Ratios}

One means of assessing whether the nebular calculations described here bear any applicability to real GEHRs is by comparison of predicted and observed line ratios for these systems. Figure 3 shows line ratio plots selected for ease of comparison with those given in Figure 8 of the extensive study of GEHRs by McCall et al. (1985); the plotted points represent observational measurements from McCall et al. The plotted curves show the prediction results for a dust-free nebula (solid curves) and for a depleted nebula with full grain physics (dotted curves), as a function of changing $Z$, for the two choices of $T_{\star}$. The behavior of the oxygen lines suggests that a higher $T_{\star}$ is required at lower $Z$, in agreement with previous work (e.g., McCall et al. 1985).

Figure 3 shows that in the high metallicity regime $\left(Z \gtrsim \mathrm{Z}_{\odot}\right)$, the calculations for the undepleted nebula tend to underpredict emission in $[\mathrm{NII}]$ and $[\mathrm{SII}]$, while the flux in these lines is excessive in the calculations with grains. This pattern is more readily seen in Figure 4, which plots $[\mathrm{O} \mathrm{III}] / \mathrm{H} \beta$ as a function of $[\mathrm{N} \mathrm{III}] / \mathrm{H} \alpha$ and $[\mathrm{S} \mathrm{II}] / \mathrm{H} \alpha$ for the same data sets. A simple explanation for this behavior might be that the assumed grain component is excessive, and the actual depletion and grain effects are somewhat reduced. Other explanations are possible, however. The strength of [S II] emission is particularly sensitive to the value of $U$ (e.g., Skillman 1989), and additional calculations indicate that the predicted $[\mathrm{S} \mathrm{II}] / \mathrm{H} \alpha$ ratio for the dusty nebula can be brought into agreement with the observations by increasing $U$ by a factor of $\sim 2-3$. This remedy is less successful for the [N II] line strength, however, and fine-tuning of other parameters such as $\mathrm{N}$ abundance is likely to be necessary to bring predictions for [N II] into agreement with the observations. Decreasing $U$ in the dust-free case does not significantly increase [S II], and leads to substantial decreases in the strength of the other optical forbidden lines considered here. 
The predictions in Figure 4 permit an interesting comparison with similar plots in Figures 7 and 8 of Kennicutt, Keel, \& Blaha (1989) for observations of H II regions in the nuclei of galaxies. Kennicutt et al. (1989) noted that the nuclear sources show a systematic trend of greater [N II] and $[\mathrm{S} \mathrm{II}]$ line strength at a given value of $[\mathrm{O} \mathrm{III}] / \mathrm{H} \beta$ than is seen in extranuclear GEHRs. Their observational results for the nuclear sources are, in fact, in relatively good agreement with the predictions for dusty nebulae presented here, for $Z$ up to $\sim 2 \mathrm{Z}_{\odot}$. Kennicutt et al. suggested that the trend in the nuclear H II regions might stem from the presence of weak active nuclei that are spatially unresolved from normal circumnuclear star-forming regions. The present results suggest a possible alternative explanation whereby the enhanced [N II] and [S II] emission results from an elevated dust content in nuclear nebulae relative to disk H II regions. The nucleus corresponds to the center of a galaxy's potential well, and the interstellar pressure in such an environment is expected to exceed typical disk ISM pressures by up to 2 orders of magnitude or more (e.g., Spergel \& Blitz 1992; Helfer \& Blitz 1993). Resulting enhancements in density may foster enhanced grain formation and growth, consistent with local correlations between density and depletion, thus providing a physical motivation for why nuclear nebulae could be unusually dusty.

\subsection{Observable Extinction}

If grains are important in modifying the overall behavior of an H II region, we might expect this fact to be signaled observationally by signatures of reddening or extinction. The extinction optical depth produced by dust associated with the H II scales as in equation 2, but with optical depth at $V\left(\tau_{V}\right)$ reduced by a factor of $\sim 5$ from $\tau_{E U V}$ (Martin \& Rouleau 1989), such that $\tau_{V}$ will be only a few tenths as the dust column becomes optically thick at ionizing energies.

If the dust optical depth is increased beyond this level, the fact that the grain EUV and optical opacities are coupled implies an additional feedback mechanism that will limit optical indications of extinction. Significant $\tau_{E U V}$ reduces $N_{H}$; but achieving a large $\tau_{E U V}$ requires a substantial $N_{H}$ for typical dust-to-gas ratios. Large EUV opacity and hence significant $\tau_{V}$ will thus result only from extreme nebular conditions. For example, if internal dust is sufficient to produce $\tau_{V} \approx 1$ and hence $\tau_{E U V} \approx 5$, then $N_{H}$ (which is proportional to $\left[Q(H) n \epsilon^{2}\right]^{1 / 3}$ in the dust-free case) must be $\sim 3 \times 10^{20}$ for $Z=\mathrm{Z}_{\odot}$ (cf. eqn. 1 ). But $Q(H)$ seen by the gas will be reduced as $\tau_{E U V}$ increases and a growing fraction of the ionizing radiation is absorbed by grains; $\tau_{E U V} \approx 5$ will result in a reduction of the nebular radius by a factor of $\sim 2$ relative to the dust-free $R_{s}$, with $\sim 90 \%$ of the ionizing photons absorbed by dust for a standard dust-to-gas ratio (Petrosian, Silk, \& Field 1972; see also Mathis 1971 and Aannestad 1989). In order to obtain the $N_{H}$ producing $\tau_{V} \approx 1$, the product $\left[Q(H) n \epsilon^{2}\right]^{1 / 3}$ and hence $U$ corresponding to the dust-free case must be increased by approximately an order of magnitude.

Conditions in which dust so strongly modifies the nebula are probably only present in systems that are clearly distinct from classical GEHRs. One such class of objects is that of ultracompact 
H II regions (e.g., Wood \& Churchwell 1989), which are far denser than typical GEHRs and show observational evidence for substantial dust absorption of ionizing radiation. Similar conditions may also obtain in the dense central regions of starburst galaxies. The high $n_{e}$ and large $U$ that describe these environments are inconsistent with optical line ratios for GEHRs.

Substantial extinction may still be present with less dramatic effects on the nebula if the ionization is produced by distributed sources rather than a central compact star cluster. An idealized picture illustrating this point would be a collection of Strömgren spheres aligned along the line of sight; $\tau_{V}$ through a single sphere may be small, but the collective effect of multiple foreground systems could produce significant extinction of emission produced in the more distant spheres.

Observations suggest, however, that internal optical extinction in GEHRs is indeed fairly small. Studies of the stellar population in 30 Doradus (Malamuth \& Heap 1994) and in NGC 3603 (Moffat, Drissen, \& Shara 1994), for example, demonstrate that the massive stellar component that dominates production of ionizing radiation is relatively concentrated, suggesting that idealization of the nebula as a single Strömgren sphere is unlikely to be grossly in error. Empirical estimates of $A_{V}$ for GEHRs are also rarely more than $A_{V} \approx 1$ (Kennicutt 1984). While extinction values based on reddening of the Balmer lines alone may underestimate the total extinction, due to surface effects (Mathis 1970), radio measurements of GEHRs suggest that the optical estimates of total $A_{V}$ are not in error by large factors (Israel \& Kennicutt 1980; Caplan \& Deharveng 1986; van der Hulst et al. 1988; see also discussion in Shields 1990).

Although the total optical extinction in GEHRs is thus only modest, the effect of dust on the nebula can still be significant if EUV opacity is much larger than opacity at optical wavelengths. It also must be remembered that attenuation of the ionizing radiation field is not directly traced by the comparison between Balmer line and radio fluxes.

\section{Further Implications}

\subsection{Global Metallicity}

Existing abundance analyses of H II regions have relied heavily on the $R_{23}$ parameter [三 $([\mathrm{O}$ II $] \lambda 3727+[\mathrm{O}$ III $] \lambda \lambda 4959,5007) / \mathrm{H} \beta]$ as an indicator of the abundance of oxygen, which in turn is often taken as a proxy for the total metallicity (Pagel et al. 1979). This approach can be justified by the dominant role of oxygen as a coolant in typical nebulae; the calibration of $R_{23}$ as a function of $Z$ is nonetheless dependent at some level on the abundances of other coolants,

however. An important question is thus whether this calibration is significantly modified by the presence of grains and associated depletion. Another way of expressing this question is to ask 
whether the gas-phase oxygen abundance derived from $R_{23}$ can be translated into a total oxygen abundance (or $Z$ ) through simply scaling by the oxygen depletion factor.

To illustrate the effects of dust on the use of $R_{23}$ as an abundance indicator, Figure 5 displays $R_{23}$ as a function of oxygen abundance in the gas phase, for the calculations described in $\$ 2$. By comparing the curves for a given $T_{\star}$ that correspond to no dust and full dust effects, we can see that relatively small changes in $R_{23}$ result from the presence of grains and associated depletion at low $Z$. The shift in predicted $R_{23}$ due to grains at a given gas-phase abundance increases significantly at higher $Z$; at the same time, however, $R_{23}$ is becoming a steeper function of $Z$, such that a large shift in predicted $R_{23}$ propagates into a small difference in $Z$. In terms of the observationally relevant comparison, for a measured value of $R_{23}$, the uncertainty in $Z$ due to dust effects is likely to be $\lesssim 0.2$ dex for a gas-phase oxygen abundance of up to $\sim 3$ times the solar value. We conclude that the effects of grains are unlikely to introduce large errors in measurements of gas-phase oxygen abundance based on $R_{23}$. This conclusion differs somewhat from that of Henry (1993). The calibration of $R_{23}$ at high $Z$ is rather sensitive to $n_{e}$, however, due to suppression of fine-structure cooling (Oey \& Kennicutt 1993), as well as $U$, and the cumulative effects of these uncertainties cast doubt on whether a reliable calibration of $R_{23}$ versus $Z$ is possible at $Z>\mathrm{Z}_{\odot}$ (see also Mathis 1986).

\subsection{Temperature Gradients}

Temperature gradients in dust-free H II regions are determined by the efficiency of cooling mechanisms and the average heating per ionization as a function of radius. At very low $Z$, collisionally excited hydrogen Ly $\alpha$ plays an important role in cooling. The neutral fraction of hydrogen that can give rise to such emission increases monotonically with radius, so that the cooling efficiency increases with $r$, and $T_{e}$ tends to be a decreasing function of radius. At higher $Z$, collisionally excited lines of heavy elements become important for cooling, with infrared transitions playing an increasing role; important ions with ground-state fine structure giving rise to infrared emission include doubly ionized $\mathrm{O}, \mathrm{S}$, and $\mathrm{N}$, which boosts the cooling efficiency in the inner nebula where these species are abundant (Stasińska 1980). In addition, the frequency dependence of hydrogen and helium bound-free opacity guarantees that the average energy and resultant heating per ionizing photon increases with increasing radius. The combined effects are expected to produce a gradient $d T_{e} / d r>0$ in high metallicity H II regions 5 . Infrared transitions also play an important role in cooling the lower-ionization, outer portions of a nebula, particularly by emission of [Si II] $34.8 \mu \mathrm{m}$, lines of [Fe II] (e.g., see Nussbaumer \& Storey 1988), and [C II] $158 \mu \mathrm{m}$.

\footnotetext{
${ }^{2}$ The resulting lines are [O III] 51.8 and $88.4 \mu \mathrm{m}$, [S III] 18.7 and $33.5 \mu \mathrm{m}$, and [N III] $57.3 \mu \mathrm{m}$.

${ }^{3}$ At very low $Z$ the gradient in heating stemming from bound-free opacity is overwhelmed by the role of Ly $\alpha$ cooling in determining the equilibrium $T_{e}$, which consequently decreases with $r$.
} 
Grains and associated depletion may have an important role in modifying the thermal structure of GEHRs beyond a simple global increase in $T_{e}$, when compared with the dust-free case. Examination of the elemental depletion factors given in Table 1 indicates that depletion has a much stronger influence on elements dominating fine-structure cooling in the low-ionization, outer portions of a nebula $(\mathrm{Si}, \mathrm{Fe}, \mathrm{C})$ than on those important in the inner nebula $(\mathrm{O}, \mathrm{S}, \mathrm{N})$. Depletion will consequently tend to steepen temperature gradients from the undepleted case, by preferentially elevating the temperature of the outer nebula. This steepening will be mitigated, however, through heating of the inner nebula by grains formed from the depleted elements. As noted in $\S 3$., the rate of grain heating will tend to scale locally with the flux of ionizing radiation. Radial dilution and attenuation of the radiation produced by a central star or stars will thus lead to a radial falloff in grain heating within a nebula, assuming a uniform distribution of dust.

These consequences can be seen quantitatively in the middle panels of Figures 6 and 7, which show $T_{e}$ profiles for the calculations described in $\S 2$., for the case of $Z=\mathrm{Z}_{\odot}$. The results of adding the effects of dust incrementally are illustrated by the successive curves. For comparison, the lower panels show the characteristic ionization state of the gas as a function of radius, as described by the ionization fraction of $\mathrm{O}^{+}$and $\mathrm{O}^{++}$. Grain heating and cooling as a function of $r$ is illustrated in the top panels. Grain heating may exceed $70 \%$ of the total heating rate in the inner nebula, and decreases monotonically with increasing radius through most of the H II region. Near $R_{s}$, the fractional heating may again become large; in this partially ionized zone, hydrogen bound-free heating is rapidly diminishing, but photons with energy $8 \lesssim h \nu<13.6 \mathrm{eV}$ continue to produce significant grain photoelectrons. Fractional cooling by dust is lower than fractional heating at all radii, with the possible exception of the outermost portions of the Strömgren sphere. These results show a general similarity to calculations with a blister geometry described by Baldwin et al. (1991).

The radial dependences of depletion and grain heating propagate into the predicted fluxes of optical lines, as can be seen by a review of Figures 1 and 2. For both choices of $T_{\star}$, emission in [O II], [S II], and [N II] (which represent relatively weakly ionized species) show a strong response to depletion, while the response in [O III] is less dramatic. In contrast, [O III] shows a much greater sensitivity to grain heating than do [O II], [S II], or [N II], for the nebula powered by the cooler star. The differences in the behavior of [O III] between the two choices of $T_{\star}$ reflect the relative extent of the $\mathrm{O}^{++}$zone (cf. Figs. 6 and 7 ). The cooler star produces a more concentrated region of $\mathrm{O}^{++}$, which is consequently more strongly affected by grain heating; the $\mathrm{O}^{++}$zone produced by the hotter star overlaps more broadly with zones of low-ionization species, introducing a greater sensitivity to depletion.

Grain heating might be expected to produce very strong enhancements of high-ionization lines that form close to the ionizing stars. In the present calculations, however, an easily observable signature of this effect is not predicted, because such lines are relatively weak, even when dust heating is taken into account. A tradeoff results in that a strong dust signature may be more apparent when $T_{\star}$ is relatively low, due to the fact that the emitting volume of a high-ionization 
species is then highly concentrated to the region of strong heating by grains; but when this volume is small, emission is likely to be weak except for a tracer with high abundance, such as oxygen. An illustration is provided in the case of $T_{\star}=38,000 \mathrm{~K}$ by [Ne III] $\lambda 3869 / \mathrm{H} \beta$, which increases by a factor of $\sim 2$ at $Z=\mathrm{Z}_{\odot}$ and by an order of magnitude at $Z=2 \mathrm{Z}_{\odot}$ when grain heating and cooling is introduced to the calculation; but invariably the [Ne III] line flux is less than $1 \%$ of that of $\mathrm{H} \beta$. If $T_{\star}$ is as high as $45,000 \mathrm{~K}$, the [NeIII] line becomes more readily observable ([Ne III $] / \mathrm{H} \beta=0.1-0.2$ ), but shows only a weak sensitivity to dust thermal effects since the $\mathrm{Ne}^{++}$ volume extends over a larger fraction of the nebula, including regions where grain photoelectric heating is relatively unimportant.

Signatures of radially dependent grain heating may be more readily detected in planetary nebulae, which can span a broader range of ionization than is typically found in H II regions. Keyes, Aller, \& Feibelman (1990) performed a detailed analysis of the spectrum of the planetary nebula NGC 7027, and noted a persistent difficulty in accounting for the observed (strong) emission in $[\mathrm{NeV}] \lambda \lambda 3346,3426$ after adjusting model nebular parameters so as to reproduce correctly the strength of He II lines. Since the He II features are recombination lines while [Ne V] is collisionally excited, the unexpected strength of the latter emission might be explained by a thermal mechanism such as grain heating in the inner nebula, if a fraction of the dust survives in this environment. Test calculations with model parameters similar to those employed by Keyes et al. are consistent with this interpretation. Detailed study of other sources is needed in order to ascertain whether grain heating is often an important influence on the emergent spectrum of planetary nebulae.

Abundance analyses based on H II region spectra are potentially sensitive to the details of nebular temperature gradients. The derivation of an ionic abundance from a collisionally excited emission line requires the use of a $T_{e}$ value appropriate to that ion. Nonuniform temperature structure necessitates the use of different characteristic temperatures for different ions; in addition, temperature-sensitive (auroral/nebular) line ratios of a single ion can differ from ion-weighted temperatures that are physically appropriate for extracting abundance information from line strengths. Radial gradients are a specific case of temperature fluctuations that contribute lingering uncertainty in the calibration of abundances based on nebular studies (e.g., Peimbert 1967; Peimbert, Storey, \& Torres-Peimbert 1993). Detailed aspects of these problems have been reviewed by Garnett (1992), who derived theoretical relations between ion-weighted temperatures for various species, as well as between line-ratio and ion-weighted temperatures. Garnett's models do not include the consequences of grains, although he notes their possible significance for perturbing nebular temperature structure.

The behavior of ion-weighted temperatures in the present calculations suggest some possible modifications to previous theoretical work. Garnett's models included depletion of Si (which he excluded completely) and Fe, and our calculations with only depletion are consistent with his results. In the preceding discussion, it was noted that depletion selectively boosts $T_{e}$ in the outer nebula, while grain heating is most important in the inner nebula. As a result, models that include 
depletion but neglect the thermal effects of the corresponding grain population may overestimate the steepness of the temperature gradient in an enriched nebula. These expectations are borne out in part in the present calculations, as can be seen by examination of Figures 8 and 9, which plot the ion-weighted temperatures $T\left(\mathrm{O}^{+}\right)$and $T\left(\mathrm{~S}^{++}\right)$as functions of $T\left(\mathrm{O}^{++}\right)$. These temperatures are calculated according to equation (1) in Garnett (1992); the plotted points span $Z=0.1-3$ $\mathrm{Z}_{\odot}$. Also shown in these figures are the trends found by Garnett (and confirmed by us) for a nebula with depletion alone, i.e., his equations (2) and (3), as well as diagonal lines of uniform $T_{e}$.

At high $Z$, the calculations with depletion and grain effects show a more nearly isothermal behavior than predictions restricted to depletion, for the case of the cooler $T_{\star}$. For comparison, the predictions for a dust-free and undepleted nebula are also shown; interestingly, these predictions are in better agreement with the calculations with full dust effects than with those taking only depletion into account. This behavior reflects a rough tendency for depletion and grain heating to balance each other in terms of their net effect on $d T_{e} / d r$. For the hotter $T_{\star}, T\left(\mathrm{~S}^{++}\right)$at a given $T\left(\mathrm{O}^{++}\right)$for the dusty nebula is still somewhat overpredicted by the relation with depletion only, while $T\left(\mathrm{O}^{+}\right)$versus $T\left(\mathrm{O}^{++}\right)$is consistent with the depletion-only results. For $T_{\star}=45,000$ $\mathrm{K}, \mathrm{S}^{++}$occupies an intermediate zone in radius that experiences a smaller relative increase in $T_{e}$ than either of the inner or outer regions weighted respectively by $\mathrm{O}^{++}$or $\mathrm{O}^{+}$; the overall gradient in $T_{e}$ remains positive for the most part, however, such that the relation between $T\left(\mathrm{O}^{++}\right)$ and $T\left(\mathrm{O}^{+}\right)$does not change appreciably from the dust-free or depletion-only case. Calculations with $n_{e}$ increased to 100 and $1000 \mathrm{~cm}^{-3}$ produce a general increase in nebular temperatures, due to partial collisional suppression of fine-structure cooling, but the ion-weighted temperature behavior is qualitatively the same as seen in Figures 8 and 9. These results also generally show a weak sensitivity to $U$. Increasing $\epsilon$ such that $\log U=-2.5$ causes the dust-free calculations for $T_{\star}=38,000 \mathrm{~K}$ to approach Garnett's relations, since the inner zone of high-ionization that cools efficiently expands, resulting in a steeper $T_{e}$ gradient; the results for the dusty nebula are qualitatively unchanged, however. We find reasonable agreement in the present calculations with relations listed by Garnett for other ion-weighted temperatures [i.e., $T\left(\mathrm{Ar}^{++}\right)=T\left(\mathrm{~S}^{++}\right)$; $\left.T\left(\mathrm{~N}^{+}\right)=T\left(\mathrm{O}^{+}\right) ; T\left(\mathrm{~N}^{++}\right)=T\left(\mathrm{O}^{++}\right) ; T\left(\mathrm{Ne}^{++}\right) \approx T\left(\mathrm{O}^{++}\right) ; T\left(\mathrm{~S}^{+}\right) \approx T\left(\mathrm{O}^{+}\right)\right]$.

If enriched GEHRs contain significant quantities of dust, the present results suggest that these nebulae may be treated as isothermal systems in terms of the relative behavior of ion-weighted temperatures, to good approximation. While this statement is strictly true only for the calculations presented here with the cooler $T_{\star}$, empirical evidence suggests that the characteristic $T_{\star}$ of a nebula decreases with increasing metallicity, such that a relatively soft radiation field is, in fact, appropriate for enriched systems with $T_{e} \lesssim 7000 \mathrm{~K}$. If dust is efficiently destroyed in H II regions, an isothermal treatment may still be appropriate, since the effects of depletion as well as grain heating would be vitiated.

\footnotetext{
${ }^{4} \mathrm{~A}$ relevant technical point is that Cloudy employs a modified on-the-spot (OTS) approximation for transfer of the hydrogen and helium diffuse radiation field. In some cases OTS can lead to an estimated temperature gradient
} 
We note that nebular models with depletion alone may nonetheless produce accurate predictions if grains are preferentially destroyed at small radii. In this situation the effects of grain heating that predominate at small $r$ would be removed, while depletion effects that selectively influence the outer nebula would still be relevant. Evidence for reduced depletion in the inner, high-ionization regions of planetary nebulae has been discussed recently by Kingdon, Ferland, \& Feibelman (1995).

Modifications to relations between line-ratio-inferred $T_{e}$ and ion-weighted temperatures will also result from dust effects. In the present calculations the largest discrepancies between the two temperature values occur at ion-weighted values of $T_{e} \lesssim 6000 \mathrm{~K}$; in this domain, auroral transitions employed for direct estimation of $T_{e}$ are generally too weak to be observable. Additional perturbations in temperature estimates from line ratios (and also ion-weighted $T_{e}$ values) may result from more complicated spatial distributions of dust, as noted by Peimbert et al. (1993).

As a final cautionary note, we remind the reader that the present calculations (and many others in the literature) assume a constant density, independent of $r$. While a detailed inquiry into the effects of nonuniform density is beyond the scope of the present work, line fluxes and temperature structure can certainly deviate in important ways from the predictions given here if large gradients in density exist within a nebula. Observations suggest that $n_{e}$ is often a decreasing function of $r$ in GEHRs (e.g., Kennicutt 1984). For H II regions with gradients of this form, the temperature of the inner nebula may be boosted and positive gradients in $T_{e}$ again diminished if $n_{e}$ at small radii is sufficient to partially suppress cooling via infrared fine-structure transitions with low critical densities. In general terms, however, the physical effects of the generic grains discussed in this paper are independent of the density structure of the nebula in which the dust resides.

\section{Conclusions}

Embedded dust can modify the optical spectrum of GEHRs, through a combination of effects that mostly act to increase $T_{e}$. The largest perturbations are generally the result of depletion of coolants from the gas phase, although grain opacity and heating may be significant in some cases. The net result is usually an increase in optical forbidden-line strengths, particularly at high metallicity. Dust may lead to nonnegligible modification of nebular behavior without introducing strong signatures of reddening or extinction.

that is too steep compared to an outward-only approximation (which was employed by Garnett 1992) or a rigorous treatment of radiative transfer (i.e., $d T_{e} / d r$ is too large; see, for example, discussion in Sarazin 1977; McCall et al. 1985; Ferland et al. 1995), which suggests that our results are conservative in that we are unlikely to predict a (positive) $d T_{e} / d r$ that is too shallow. 
The influence of grains provides a simple way of explaining the observational lack of H II regions emitting a pure Balmer-line spectrum at optical wavelengths, which is expected on theoretical grounds for dust-free nebulae with metallicity only slightly greater than $\mathrm{Z}_{\odot}$. At high $Z$, predicted enhancements of $[\mathrm{S} \mathrm{II}]$ and $[\mathrm{N} \mathrm{II}]$ in particular show a resemblance to the empirical behavior of $\mathrm{H}$ II regions in galaxy nuclei. If these environments are particularly dusty, due perhaps to characteristically elevated densities, the influence of dust provides an alternative mechanism to weak active nuclei for explaining the anomalous line ratios of these sources.

Depletion and heating by photoelectrons emitted by grains in H II regions may introduce significant modifications to nebular temperature structure. Depletion selectively influences elements that are important for cooling the outer portions of nebulae, while grain heating is most effective at small radii. Modifications to temperature gradients within GEHRs by dust may necessitate changes in assumptions employed in nebular abundance analyses, specifically in the relationships between ion-weighted temperatures for different species. For chemically enriched GEHRs, the present results suggest that these nebulae can be treated as nearly isothermal systems.

Inclusion of grain physics is unlikely to result in large changes of gas-phase heavy-element abundances derived for low- $Z$ objects and GEHRs ionized by relatively hot stars. In general, dust-related phenomena will contribute an uncertainty of $\sim 0.2$ dex or less in gas-phase oxygen abundances derived from the $R_{23}$ parameter. Modifications to nebular temperature structure by dust and associated depletion may be significant in H II regions described by high $Z$ and low $T_{*}$, and should be considered when deriving relative abundances for heavy elements.

This work was supported by NASA through grant number HF-1052.01-93A from the Space Telescope Science Institute, which is operated by the Association of Universities for Research in Astronomy, Inc., under NASA contract NAS5-26555. Additional support was provided by the National Science Foundation through grants AST-9019150 and AST-9421145. We thank Gary Ferland for conversations that stimulated this work, as well as for access to Cloudy. Informative discussions with John Black, Don Garnett, Sally Oey, and Evan Skillman are gratefully acknowledged. We also thank the anonymous referee for helpful comments on the manuscript. 
TABLE 1

Relative Abundances ${ }^{\mathrm{a}}$ at $Z=\mathrm{Z}_{\odot}$

\begin{tabular}{lrc}
\hline \hline Element & Total & Depleted \\
\hline $\mathrm{He}$ & 10.99 & 10.99 \\
$\mathrm{C}$ & 8.56 & 8.22 \\
$\mathrm{~N}$ & 8.05 & 7.95 \\
$\mathrm{O}$ & 8.93 & 8.73 \\
$\mathrm{Ne}$ & 8.09 & 8.09 \\
$\mathrm{Na}$ & 6.30 & 5.50 \\
$\mathrm{Mg}$ & 7.58 & 7.15 \\
$\mathrm{Al}$ & 6.47 & 5.53 \\
$\mathrm{Si}$ & 7.55 & 6.55 \\
$\mathrm{~S}$ & 7.21 & 7.21 \\
$\mathrm{Ar}$ & 6.56 & 6.56 \\
$\mathrm{Ca}$ & 6.36 & 3.66 \\
$\mathrm{Fe}$ & 7.67 & 5.97 \\
$\mathrm{Ni}$ & 6.25 & 4.26 \\
\hline
\end{tabular}

${ }^{a}$ Abundances are logarithmic +12 , by number relative to hydrogen. 


\section{REFERENCES}

Aannestad, P. A. 1989, ApJ, 338, 162

Arsenault, R., \& Roy, J.-R. 1988, A\&A, 201, 199

Baldwin, J. A., Ferland, G. J., Martin, P. G., Corbin, M. R., Cota, S. A., Peterson, B. M., \& Slettebak, A. 1991, ApJ, 374, 580

Bohren, C. F., \& Huffman, D. R. 1983, Absorption and Scattering of Light by Small Particles (New York: Wiley)

Caplan, J., \& Deharveng, L. 1986, A\&A, 155, 297

Cowie, L. L., \& Songaila, A. 1986, ARA\&A, 24, 499

Draine, B. T. 1989, in Evolution of Interstellar Dust and Related Topics, ed. A. Bonetti, J. M. Greenberg, \& S. Aiello (Amsterdam: North Holland), 91

Draine, B. T., \& Lee, H. M. 1984, ApJ, 285, 89

Ferland, G. J. 1994, Univ. of Kentucky Phys. \& Astr. Internal Report

Ferland, G. 1995, in The Analysis of Emission Lines, ed. R. E. Williams \& M. Livio (Cambridge: Cambridge University Press), in press

Ferland, G. J., \& Truran, J. W. 1981, ApJ, 244, 1022

Garnett, D. R. 1992, AJ, 103, 1330

Garnett, D. R., Dufour, R. J., Torres-Peimbert, S., Shields, G. A., Skillman, E. D., Terlevich, E., \& Terlevich, R. J. 1995, preprint

Grevesse, N., \& Anders, E. 1989, in Cosmic Abundances of Matter, ed. C. J. Waddington (New York: AIP), 1

Habing, H. T., \& Israel, F. P. 1979, ARA\&A, 17, 345

Helfer, T. T., \& Blitz, L. 1993, ApJ, 419, 86

Henry, R. B. C. 1993, MNRAS, 261, \& Shields, G. A. 1985, ApJS, 57, 1

Hummer, D. G., \& Storey, P. J. 1987, MNRAS, 224, 801

Israel, F. P., \& Kennicutt, R. C. 1980, Ap. Lett., 21, 1

Kennicutt, R. C. 1984, ApJ, 287, 116

- 1988, ApJ, 334, 144 
Kennicutt, R. C., Keel, W. C., \& Blaha, C. A. 1989, AJ, 97, 1022

Keyes, C. D., Aller, L. H., \& Feibelman, W. A. 1990, PASP, 102, 59

Kingdon, J., Ferland, G. J., \& Feibelman, W. A. 1995, ApJ, 439, 793

Kurucz, R. L. 1979, ApJS, 40, 1

Malamuth, E. M., \& Heap, S. R. 1994, AJ, 107, 1054

Martin, P. G., \& Rouleau, F. 1989, in Extreme Ultraviolet Astronomy, ed. R. F. Malina \& S. Bowyer (Elmsford, NY: Pergamon), 341

Mathis, J. S. 1970, ApJ, 159, 263

—. 1971, ApJ, 167, 261

- 1983, ApJ, 267, 119

. 1986, PASP, 98, 995

. 1990, ARA\&A, 28, 37

Mathis, J. S., Rumpl, W., \& Nordsieck, K. H. 1977, ApJ, 217, 425

McCall, M. L., Rybski, P. M., \& Shields, G. A. 1985, ApJS, 57, 1

Melnick, J. 1979, ApJ, 228, 112

Moffat, A. F. J., Drissen, L., \& Shara, M. M. 1994, ApJ, 436, 183

Nussbaumer, H., \& Storey, P. J. 1988, A\&A, 193, 327

Oey, M. S., \& Kennicutt, R. C. 1993, ApJ, 411, 137

Oliveira, S., \& Maciel, W. J. 1986, Ap\&SS, 126, 211

Pagel, B. E. J., Edmunds, M. G., Blackwell, D. E., Chun, M. S., \& Smith, G. 1979, MNRAS, 189, 95

Peimbert, M. 1967, ApJ, 150, 825

Peimbert, M., Storey, P. J., \& Torres-Peimbert, S. 1993, ApJ, 414, 626

Petrosian, V., \& Dana, R. A. 1980, ApJ, 241, 1094

Petrosian, V., Silk, J., \& Field, G. B. 1972, ApJ, 177, L69

Rubin, R. H. 1985, ApJS, 57, 349 
Sarazin, C. L. 1977, ApJ, 211, 772

Shields, G. A. 1990, ARA\&A, 28, 525

Simpson, J. P. 1975, A\&A, 39, 43

Skillman, E. D. 1989, ApJ, 347, 883

Spergel, D. N., \& Blitz, L. 1992, Nature, 357, 665

Stasińska, G. 1980, A\&A, 85, 359

Strom, S. E., Strom, K. M., Grasdalen, G. L., \& Capps, R. W. 1974, ApJ, 193, L7

van der Hulst, J. M., Kennicutt, R. C., Crane, P. C., \& Rots, A. H. 1988, A\&A, 195, 38

Veilleux, S., \& Osterbrock, D. E. 1987, ApJS, 63, 295

Vila-Costas, M. B., \& Edmunds, M. G. 1993, MNRAS, 265, 199

Vílchez, J. M., \& Pagel, B. E. J. 1988, MNRAS, 231, 257

Wood, D. O. S., \& Churchwell, E. 1989, ApJS, 69, 831 


\section{Figure Captions}

Fig. 1 - Line ratios plotted as a function of total metallicity, with dust effects added incrementally, for $T_{\star}=38,000 \mathrm{~K}$. Solid curves: no depletion or grains; dotted: depletion only; short dashed: depletion + grain opacity; long dashed: depletion, with grain opacity, heating, and cooling. The lines $[\mathrm{O}$ II $] \equiv \lambda \lambda 3726,3729 ;[\mathrm{O} \mathrm{III}] \equiv \lambda \lambda 4959,5007 ;[\mathrm{S} \mathrm{II}] \equiv \lambda \lambda 6716,6731 ;[\mathrm{N} \mathrm{II}]$ $\equiv \lambda \lambda 6548,6583$. Approximate lower bounds to observed ratios in H II regions are indicated by the dotted horizontal lines.

Fig. $2-$ Same as Figure 1, for $T_{\star}=45,000 \mathrm{~K}$.

Fig. 3 - Loci of line ratios resulting from variation in $Z\left(=0.1-5 \mathrm{Z}_{\odot}\right.$ ), for dust-free (solid curves) and dusty (dotted curves) nebulae. Values of $Z$ are indicated by the corresponding filled points. Crosses represent measured, dereddened values for GEHRs taken from McCall et al. (1985).

Fig. 4 - Same as Figure 3, with axes chosen for comparison with results for nuclear and disk GEHRs studied by Kennicutt et al. (1989). A general discussion of the loci of emission-line nuclei and other objects in line-ratio diagrams can be found in Veilleux \& Osterbrock (1987).

Fig. 5 - Values of $R_{23}$ as a function of gas-phase oxygen abundance. Results are shown for nebular cases that are dust-free (solid curves) and dusty (dotted curves), with oxygen abundance normalized to $\log (\mathrm{O} / \mathrm{H})_{\odot}=-3.07$ by number. The upper two curves assume $T_{\star}=45,000 \mathrm{~K}$, while the lower pair assumes $T_{\star}=38,000 \mathrm{~K}$. The abundances of other heavy elements scale with oxygen as described in $\S 2$.

Fig. 6 - Radial behavior of thermal and ionization properties for $T_{\star}=38,000 \mathrm{~K}$ and $Z=$ $\mathrm{Z}_{\odot}$. Central panel: $T_{e}$ as a function of radius, with depletion and grain effects added incrementally. Top panel: heating and cooling contributed by grains as a fraction of the total. Bottom panel: Ionization fraction of $\mathrm{O}^{+}$and $\mathrm{O}^{++}$; solid curves represent a dust-free nebula, while the dashed curves show the result after adding full dust physics.

Fig. 7 - Same as Figure 6 , for $T_{\star}=45,000 \mathrm{~K}$.

Fig. $8-T\left(\mathrm{O}^{+}\right)$versus $T\left(\mathrm{O}^{++}\right)$for the dust-free and dusty calculations, where the temperatures represent ion-weighted averages. The dashed line represents the theoretical relation obtained by Garnett (1992) for depleted nebulae, while the dotted line represents the isothermal case.

Fig. $9-T\left(\mathrm{~S}^{++}\right)$versus $T\left(\mathrm{O}^{++}\right)$for the dust-free and dusty calculations. The dashed line represents the theoretical relation obtained by Garnett (1992) for depleted nebulae, while the dotted line represents the isothermal case. 


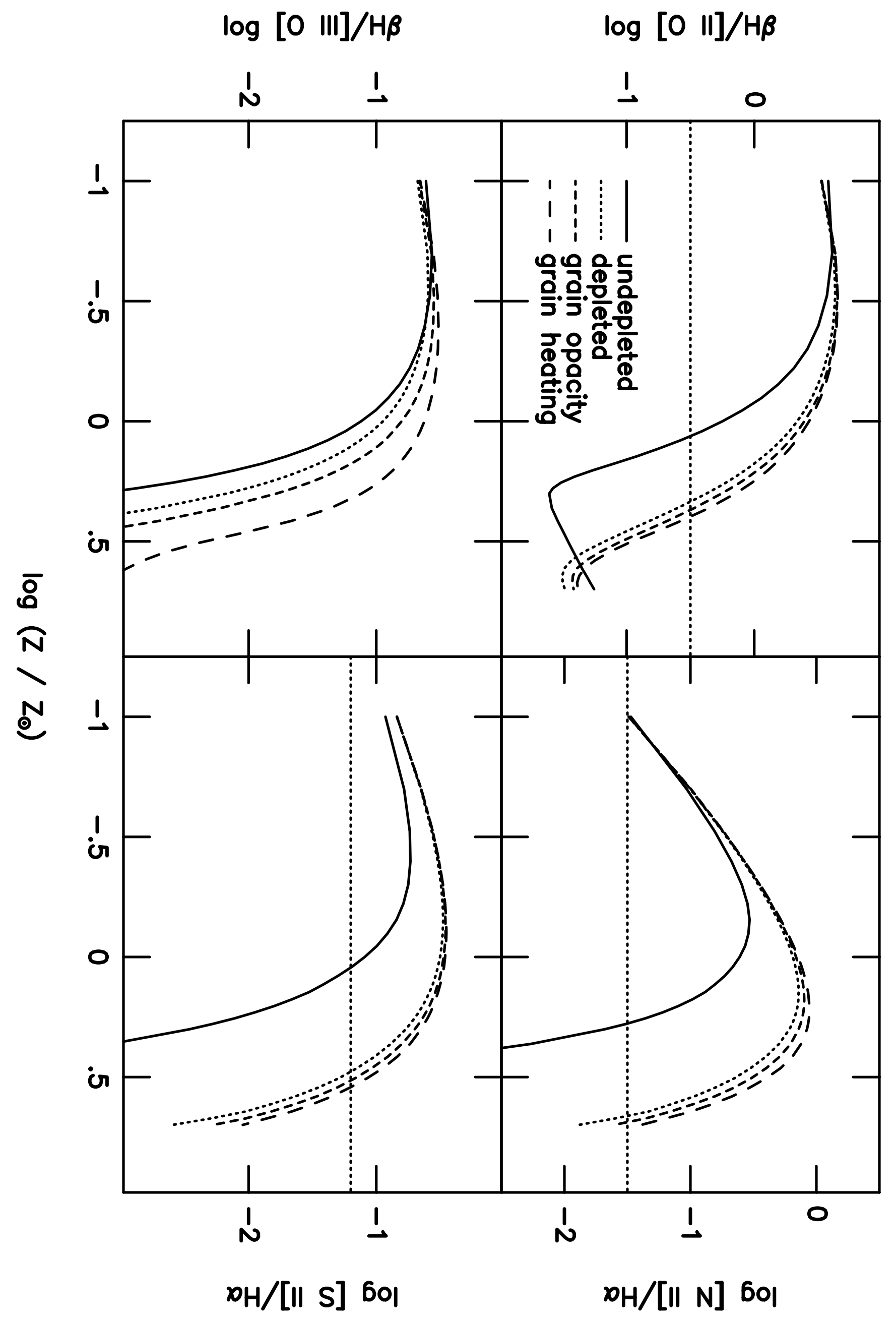




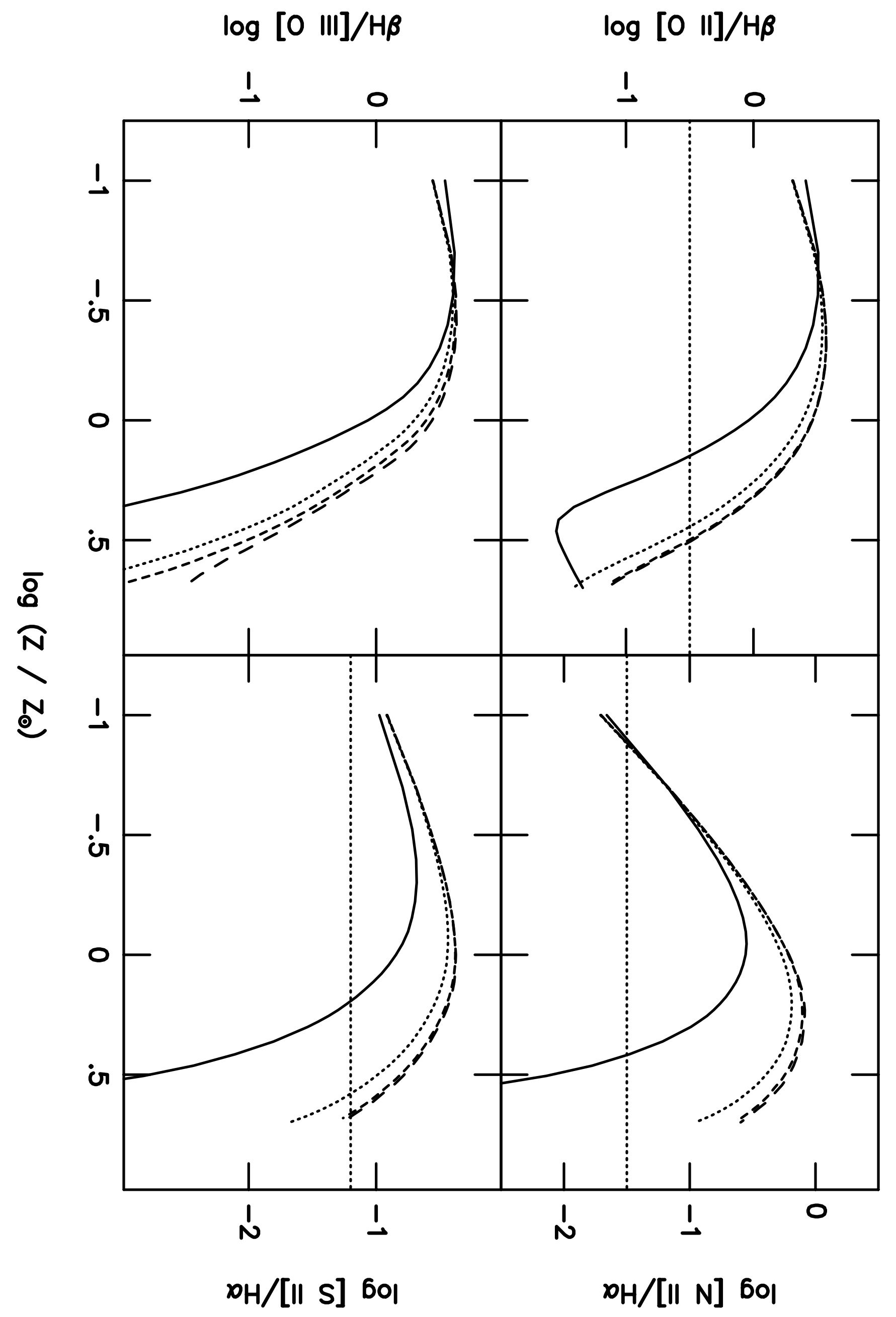




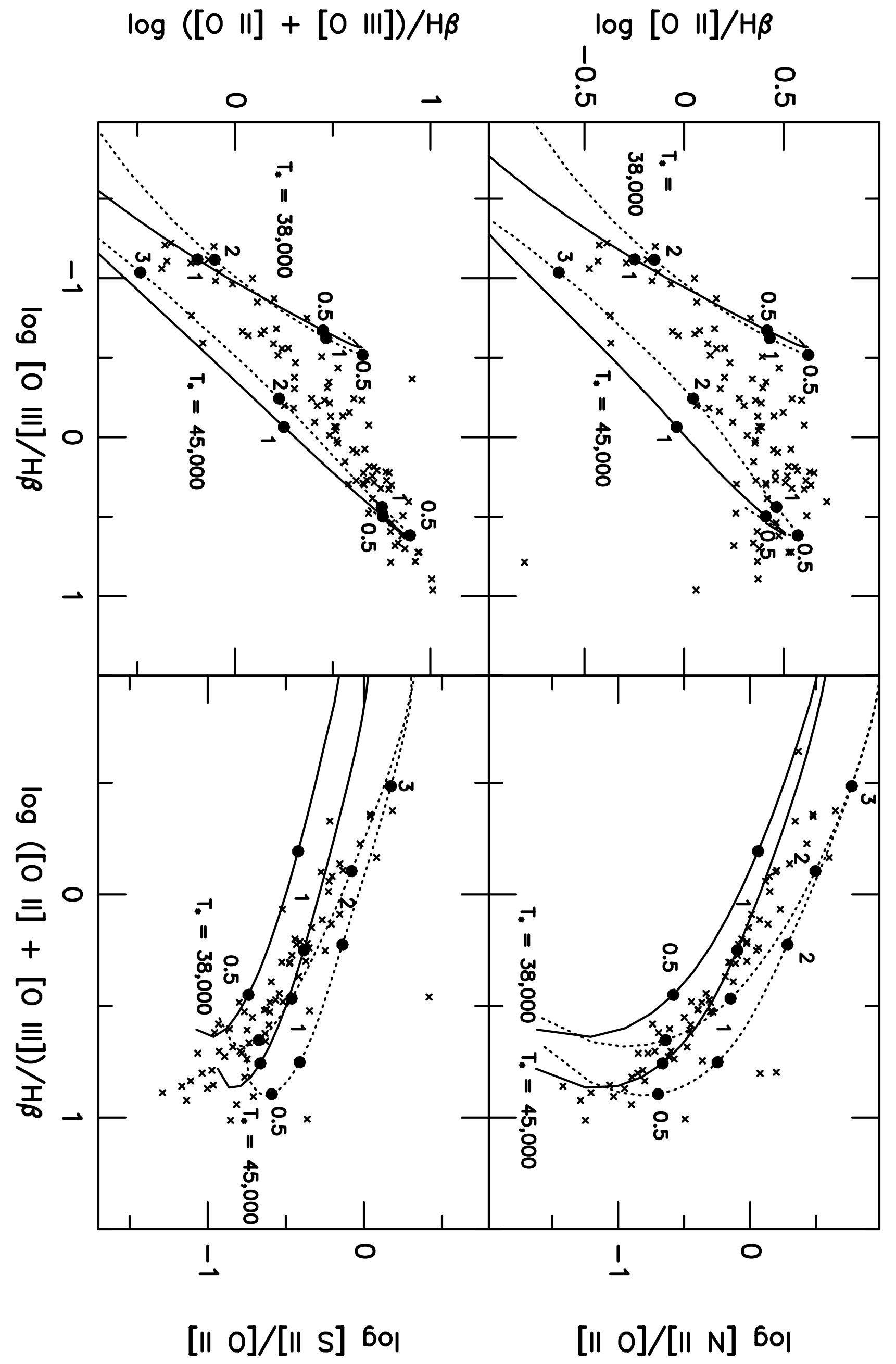


$\log \left[\begin{array}{ll}0 & I I I\end{array}\right] / H \beta$

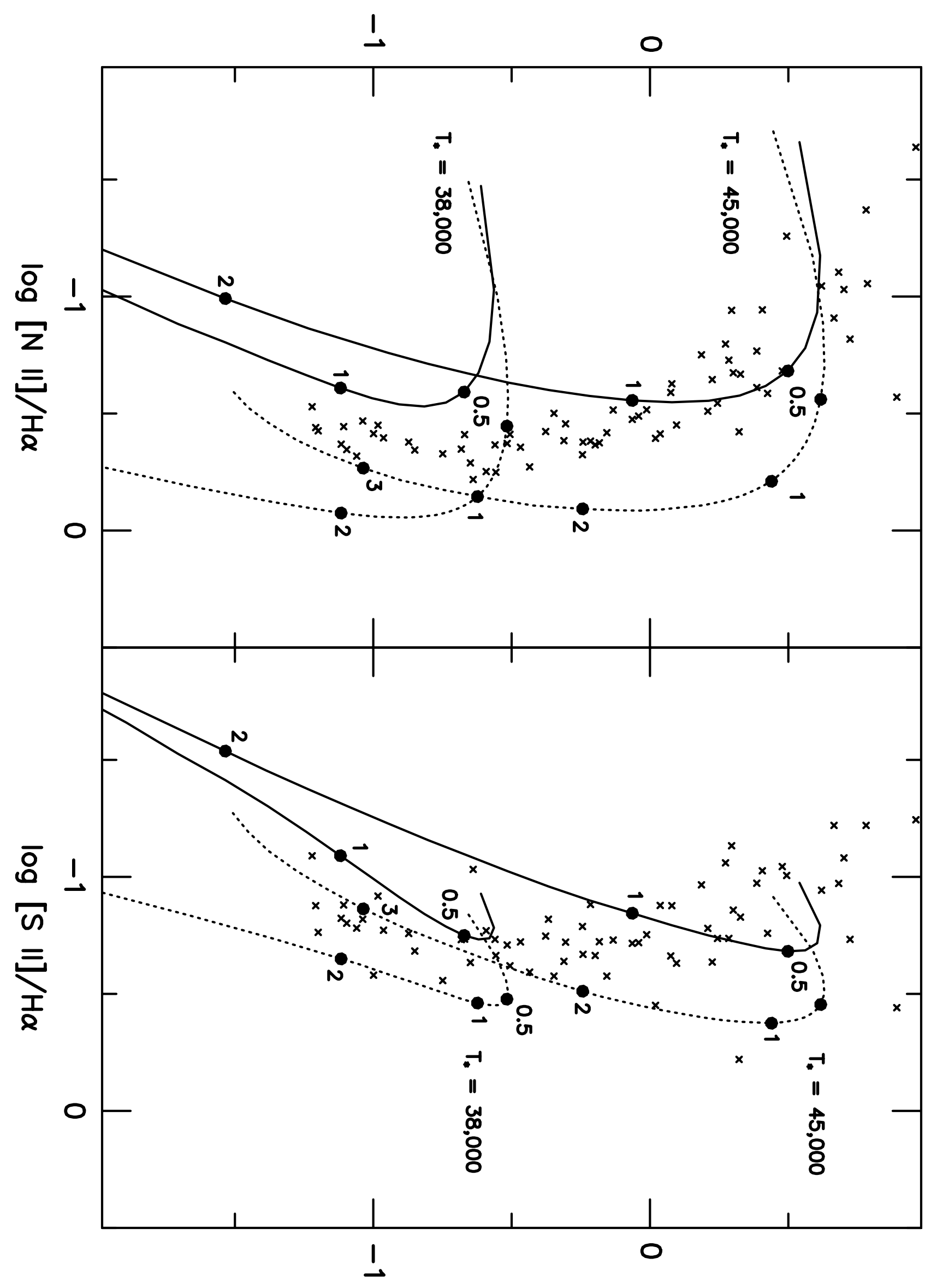




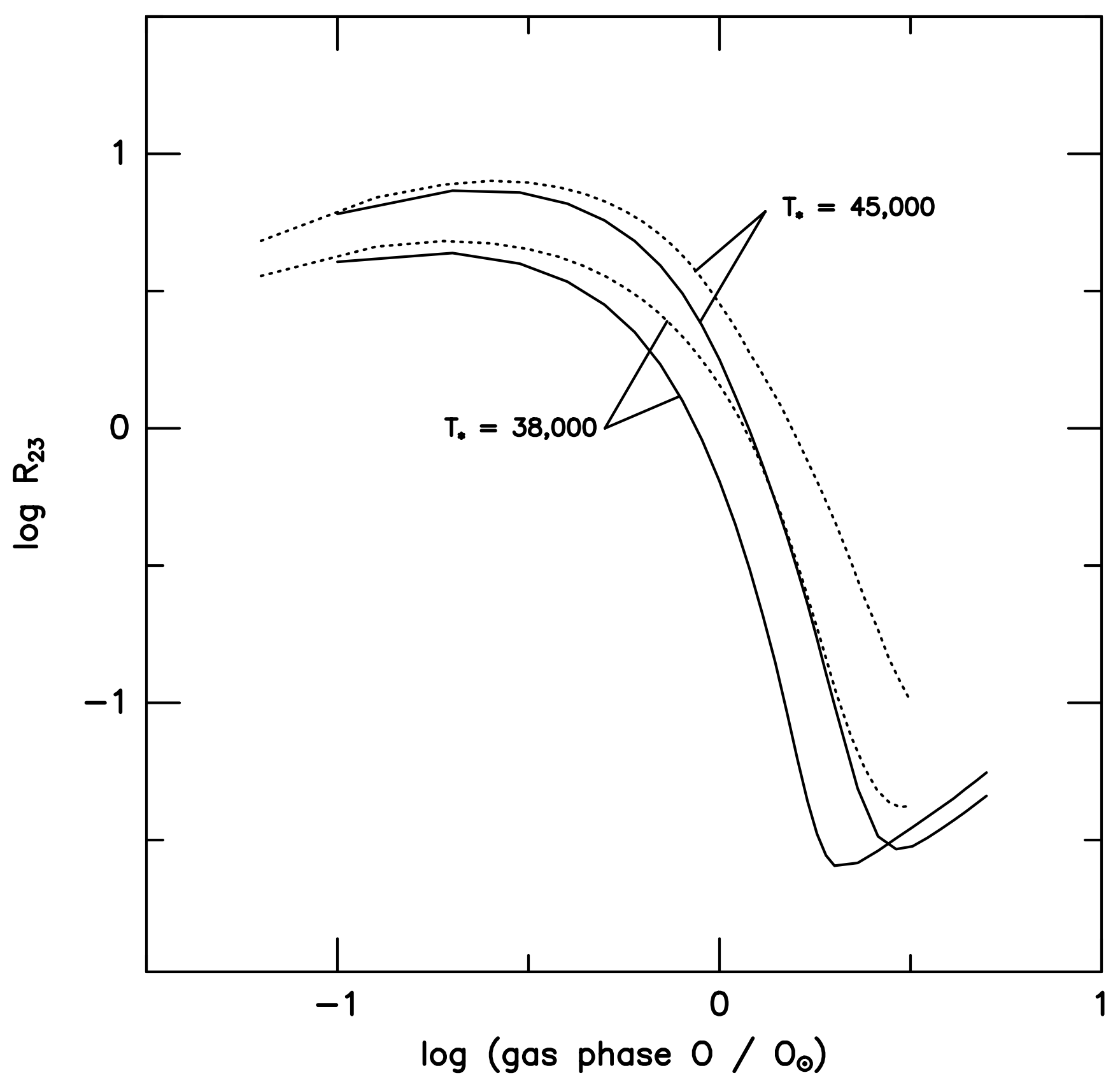




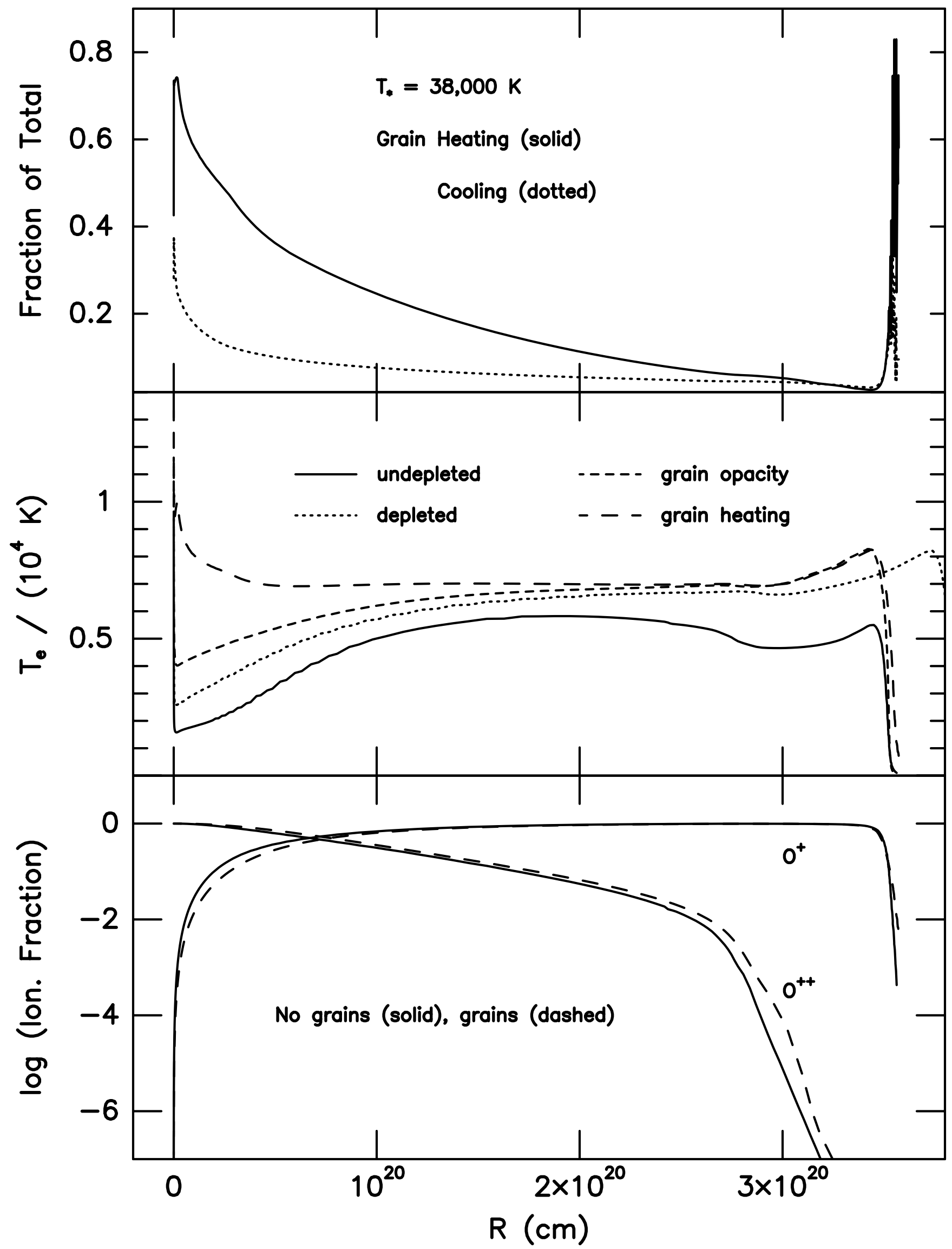




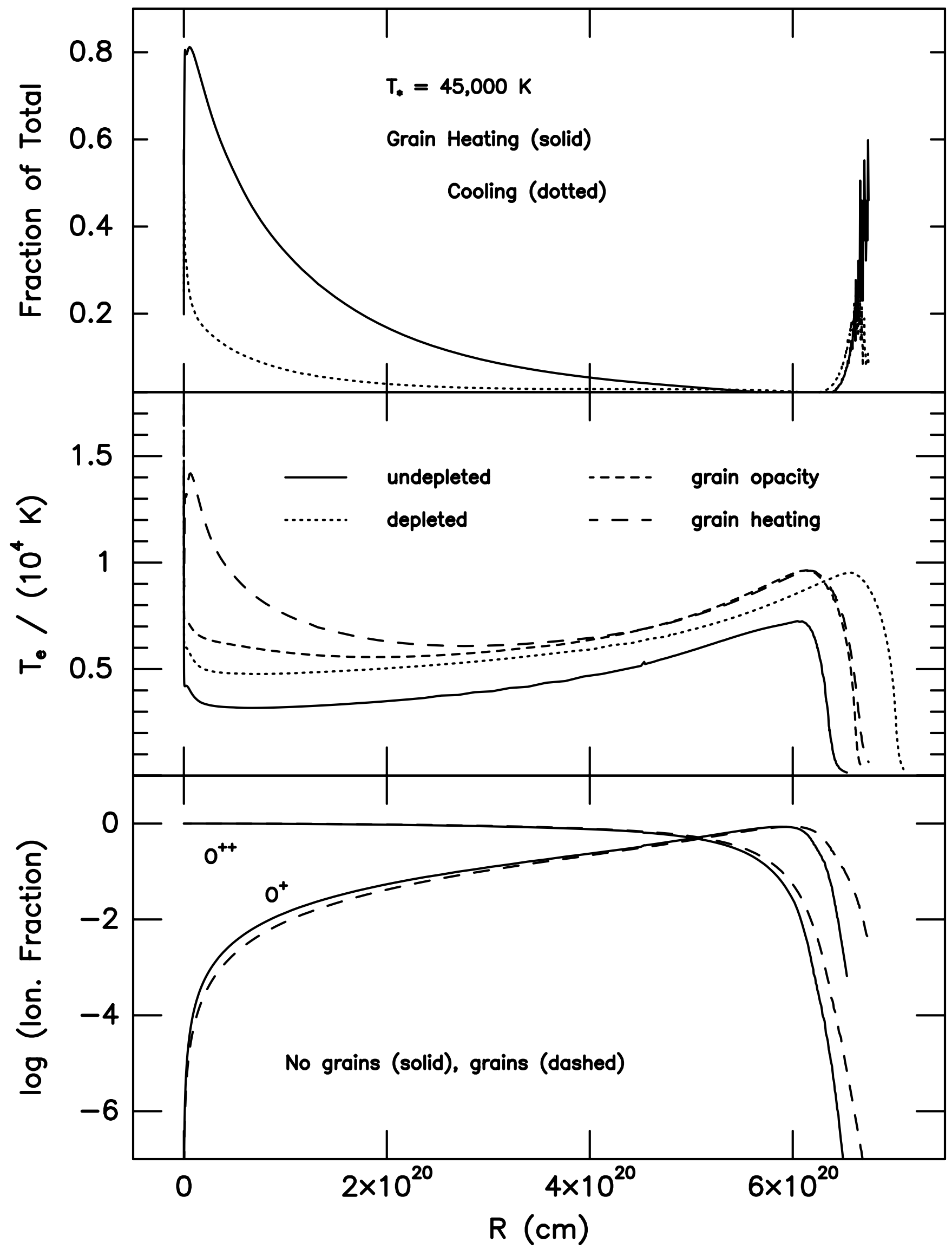




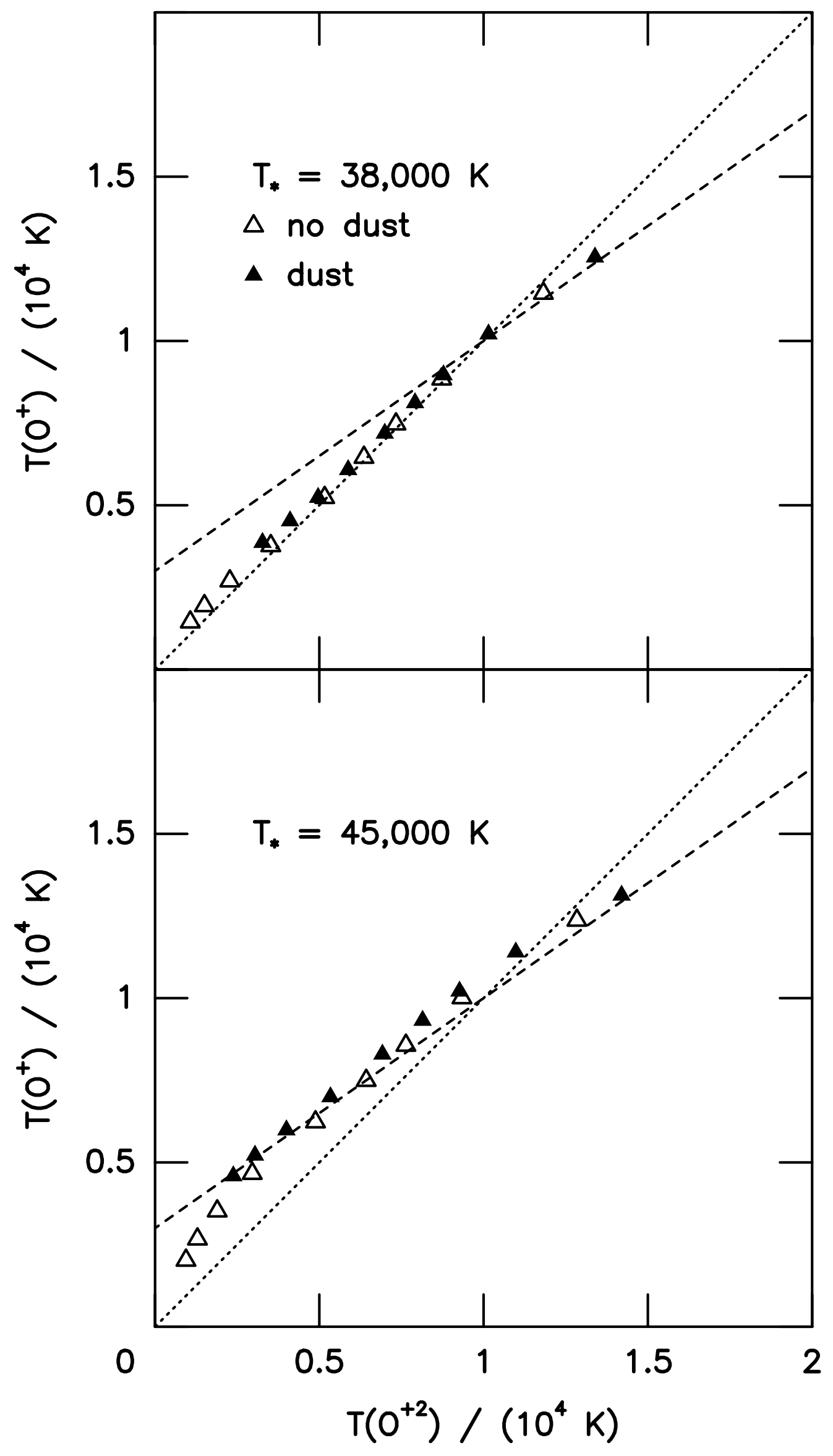




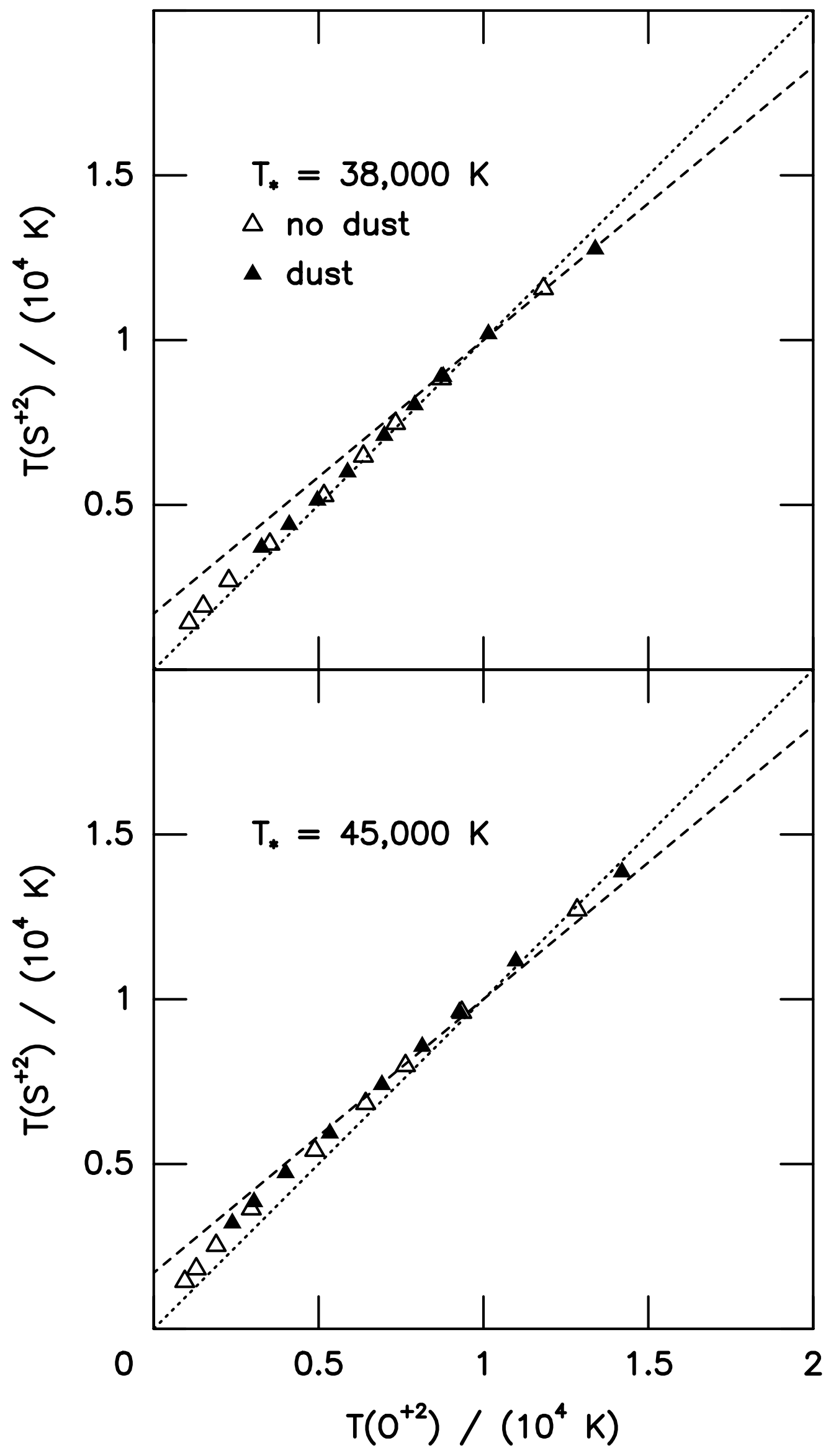

